\title{
Real-Time Fault Simulation of Mechanic-Electronic- Hydraulic System Based on Virtual Prototyping
}

\author{
Deyu He*, Niaoqing $\mathrm{Hu}$, Lei $\mathrm{Hu}$ and Ling Chen \\ Laboratory of Science and Technology on Integrated Logistics Support \\ National University of Defense Technology \\ Deya Road No.109, Changsha, Hunan, 410073, China \\ *Corresponding author
}

\begin{abstract}
Lack of historical fault data in real-time working condition is a pivotal problem in safety analysis of large-scale mechanic-electronic-hydraulic systems. To deal with this problem, this paper proposed a real-time fault injection and simulation method based on virtual prototyping. First, the virtual prototyping of actual system was built in AMESim and its credibility was verified and validated. On this basis, several typical faults were injected in the virtual prototyping. To realize real-time fault injection, this paper utilized MATLAB/Simulink to carry out Co-simulation with AMESim by designing I/O interface. Steering system was taken as case study to verify the proposed method. Simulation result showed that the acquired simulation data is credible and available.
\end{abstract}

Keywords-virtual prototyping; mechanic-electronic-hydraulic system; fault injection; real-time fault simulation

\section{INTRODUCTION}

The reliability and safety of large-scale and complicated mechanic-electronic-hydraulic systems is calling for more and more attention in modern society. Quantitative fault safety analysis of those systems is therefore very significant. However, because the actual systems are mainly working under normal condition, data under faulty condition is lacking seriously. As a result, the lack of high quality fault data, rather than the analysis methods, is account for realistic difficulty of quantitative safety analysis [1]. Therefore, the acquisition of fault data in real-time working condition is pivotal to solve this problem, which is focused by the presented paper.

Under the great promotion of modern computer technology, simulation technique has been developing significantly in the past decades. Simulation is generally studied and applied in almost every field in modern society. High efficiency, low cost and good flexibility are the outstanding advantages of simulation. As an important branch of simulation technique, virtual prototyping has been developing rapidly since 1980s. This paper utilized virtual prototyping to solve the problem stated above. Xie proposed a modeling and Co-simulation method based on AMESim and Simulink for light passenger car in 2011[2]. Authors of this paper has presented a similar fault injection and simulation method in 2014[3], where LabVIEW was utilized. Comparatively speaking, thought LabVIEW has more colorful libraries and components for control and display, MATLAB/Simulink is more powerful in data processing and analysis than LabVIEW. The purpose of this paper is to make an improvement for the previous work.

In this paper, steering system, which is a typical mechanicelectronic-hydraulic hybrid system, will be studied. First, the virtual prototyping of the system will be built on AMESim platform. Then the credibility of the prototyping will be verified and validated. On this basis, some typical faults will be injected in the validated prototyping. By designing interface, Simulink will be utilized to make Co-simulation with AMESim to realize real-time fault injection. At last, the acquired fault simulation data will be analyzed to verify its availability.

\section{VIRTUAL PROTOTYPING AND ITS VALIDATION}

\section{A. Building Virtual Prototyping}

Steering system is used for the rudder control of vessel. It consists of operating unit, amplifier, sampling unit, rudder cylinder and rudder angel feedback units, etc., which is a typical double closed-loop mechanic-electronic-hydraulic servo control system. Rudder angle is controlled by servo route to follow the order given by operator [4]. The principle of servo control is shown as Figure 1. In this paper, AMESim is chosen as the modeling/ simulation platform.

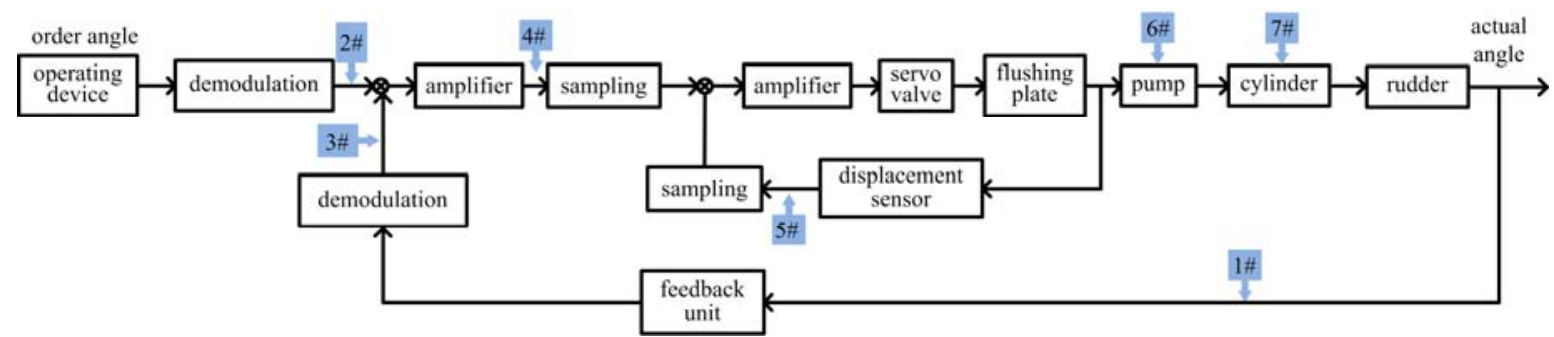

FIGURE I. CONTROL PRINCIPLE OF STEERING SYSTEM 
The established virtual prototyping is shown in Figure 2. In this prototyping, hydraulic parts correspond exactly to the actual system on component-level; however, electronic and mechanical parts simulate the actual system on the modulelevel. In this way, not only the similarity between the virtual prototyping and actual system is highly guaranteed, but also the difficulty of modeling is decreased effectively. Thus a balance can be made between the accuracy of component models and the complexity of the whole system [5]. In Figure 1 and Figure 2, measure points (MPs) $1 \# \sim 7 \#$ are marked. These MPs are used in the verification and validation $(\mathrm{V} \& \mathrm{~V})$ of virtual prototyping which is going to be stated in the next section. MP $1 \#$ is the actual angle (output angle) of steering system, with is the most important indicator that we focus. MP $2 \# \sim$ MP $5 \#$ are voltage signals in the closed loop. MP 6\# and MP 7\# are pressure signals of pump and cylinder.

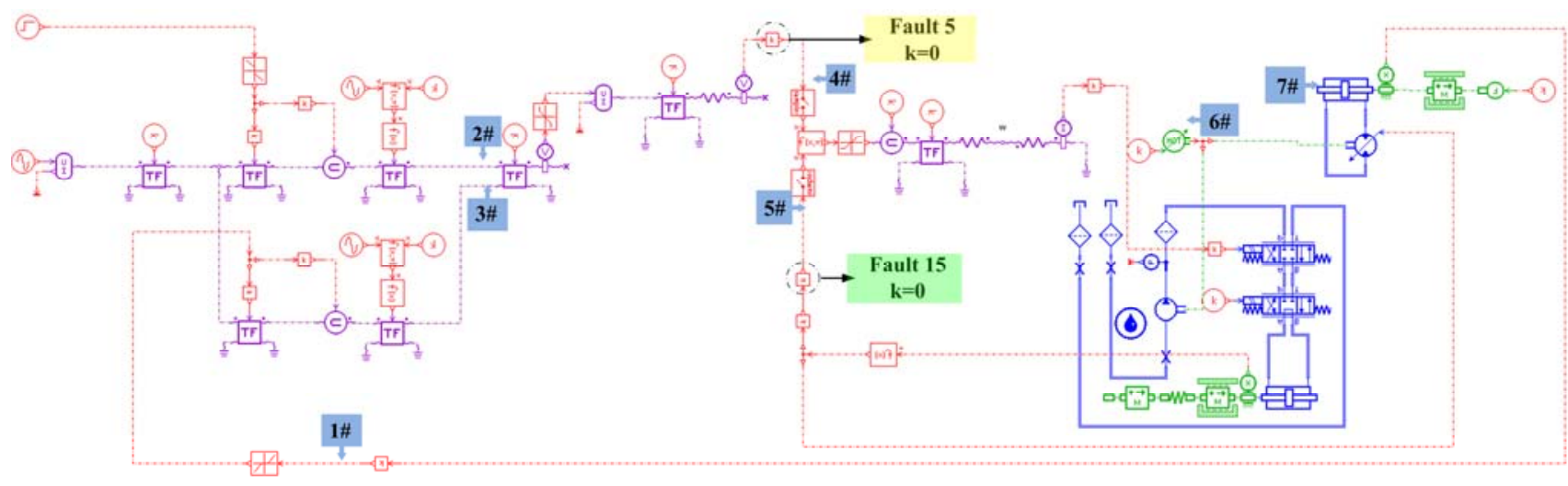

FIGURE II. VIRTUAL PROTOTYPING OF STEERING SYSTEM AND FAULT INJECTION DETAILS

\section{B. Verification and Validation of Virtual Prototyping}

In order to be used for fault injection and simulation, the established virtual prototyping needs to be verified and validated to ensure its credibility and acceptability. Detailed introduction and discussion about verification, validation and accreditation (VV\&A) could be found in [6-7]. This paper focuses on the comparison of the actual signal and simulation signal of measure points.

Suppose that $x_{t}$ and $y_{t}$ are the observation sequences of actual system and virtual prototyping respectively, thus the error sequence between them is

$$
e_{t}=x_{t}-y_{t}, t=1,2, \ldots, N
$$

where $N$ is the length of the sequence.

Root-Mean-Square $\left(\mathrm{RMS}_{\mathrm{e}}\right)$ and Correlation Coefficient $\left(\rho_{X, Y}\right)$ of the error sequence are chosen as the similarity indicator in $\mathrm{V} \& \mathrm{~V}$, which can reflect the similarity of amplitude and trend between the two sequences respectively. Their calculation formulas are shown as 2.2 and 2.3.

$$
\begin{gathered}
\mathrm{RMS}_{\mathrm{e}}=\sqrt{\frac{1}{N} \sum_{t=1}^{N} e_{t}^{2}} \\
\rho_{X, Y}=\frac{\sum_{t=1}^{N}\left(x_{t}-\bar{x}\right)\left(y_{t}-\bar{y}\right)}{\sqrt{\sum_{t=1}^{N}\left(x_{t}-\bar{x}\right)^{2} \sum_{t=1}^{N}\left(y_{t}-\bar{y}\right)^{2}}}
\end{gathered}
$$

V\&V experiments were carried out to get measured signals of MPs of actual system. The calculation results of similarity indicators are shown in Table 1, and the contrast between signals of actual system and virtual prototyping of $1 \#$ and $7 \#$ are illustrated in Figure 3. From Table 1 and Figure 3, it could be inferred clearly that the actual signal and simulation signal have a high similarity, which indicates that the virtual prototyping has a high credibility and acceptability for the use of fault injection and simulation. 


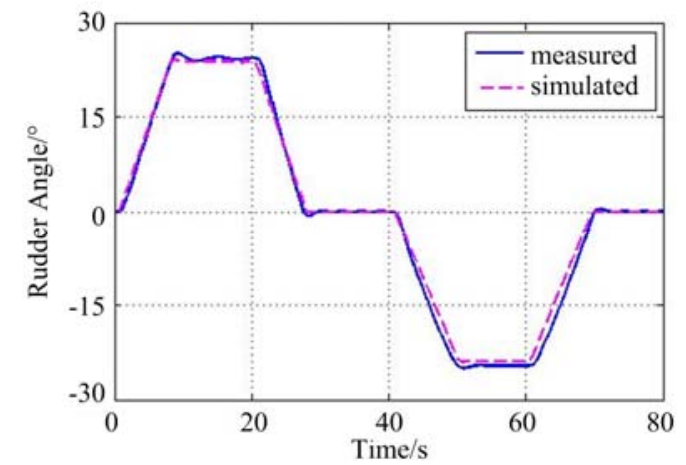

(A)

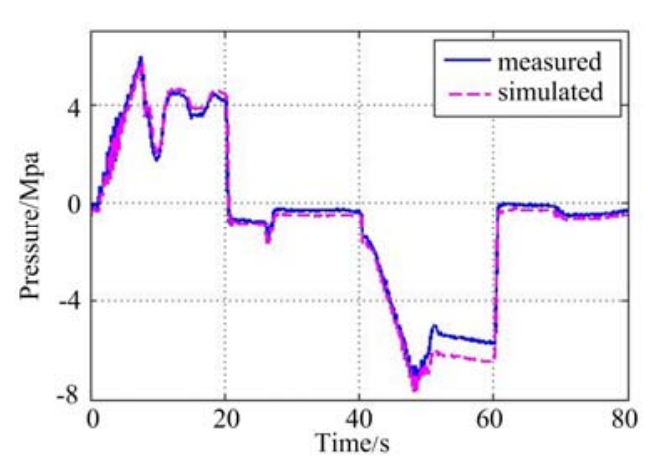

(B)

FIGURE III. CONTRAS BETWEEN ACTUAL AND SIMULATED SIGNAL: (A) SIGNAL 1\#; (A) SIGNAL 7\#

TABLE I. V\&V RESULT OF VIRTUAL PROTOTYPING

\begin{tabular}{|c|c|c|c|}
\hline $\begin{array}{c}\text { Signal } \\
\text { Index }\end{array}$ & Amplitude & $\mathrm{RMS}_{\mathrm{e}}$ & $\rho_{X, Y}$ \\
\hline $1 \#$ & $30\left(^{\circ}\right)$ & 0.89 & 0.99 \\
\hline $2 \#$ & $10(\mathrm{~V})$ & 0.27 & 0.98 \\
\hline $3 \#$ & $10(\mathrm{~V})$ & 0.07 & 0.99 \\
\hline $4 \#$ & $10(\mathrm{~V})$ & 0.52 & 0.96 \\
\hline $5 \#$ & $10(\mathrm{~V})$ & 0.35 & 0.97 \\
\hline $6 \#$ & $10(\mathrm{MPa})$ & 0.43 & 0.99 \\
\hline $7 \#$ & $10(\mathrm{MPa})$ & 0.49 & 0.99 \\
\hline
\end{tabular}

\section{TRADITIONAL FAULT INJECTION AND SimulATION}

\section{A. Fault Injection}

The core and key procedure of fault simulation is fault injection. Generally speaking, Fault injection means generating fault artificially and adding it to the studied system by building fault model according to the specified fault type and details. In this way, the fault in real system is imitated. According to the requirements of functional fault modeling and simulation in practical engineering, the fault injection in AMESim can be achieved with the following three methods[3]. Appropriate choices should be made flexibly from the above three approaches according to different fault.

\section{1) Modifying the structure of model}

The virtual prototyping of steering system consists of many different functional components. Modifying the structure of the model means adding or removing some components that are relevant to fault, and thus the originally normal model is transformed into faulty one.

\section{2) Modify the definition of the components}

This approach means changing the definition of the sub model of the AMESim graph component to generating the faulty components model.

\section{3) Modifying the critical parameters of the model}

By changing the parameters of some components that can imitate the faulty phenomenon, including the physical parameters, inputs/outputs and local variables, faulty components model can be got.

\section{B. Case Study}

As stated above, steering system is a large-scale and complicated mechanic-electronic-hydraulic hybrid system. Fault in any component of the system will lead to the whole system's failure. There are mainly 25 typical fault modes that are relatively more likely to happen. In the following part, two typical faults will be studied, namely

- Fault 5: The output of outer amplifier (MP4\#) becomes zero constantly;

- Fault 15: The feedback of flushing plate (MP5\#) becomes zero constantly.

Fault injection was conducted in the AMESim virtual prototyping. Fault injection method used here were 3) in Section 3.1. According to the characteristics of the faults, the parameters of some components that can imitate the faulty phenomenon were changed to zero, as is shown in Figure 2. Fault injection in this way can only be done before the simulation. That means fault injection is impossible in the realtime duration of a simulation.

Fault simulation is then carried out on the basis of fault injection. Step signal is chosen as the order signal. Initial value of actual angle is $30^{\circ}$. The order angle stepped to $0^{\circ}$ at 0 s. Simulation period is $10 \mathrm{~s}$ and the sample interval is $0.1 \mathrm{~s}$. The simulation results of these two faults and their comparison with normal virtual prototyping are illustrated in Figure 4. It can be inferred from the figure that fault 5 could lead to stuck rudder and fault 15 couldn't, though it seems very serious too.

Stuck rudder means the actual rudder angle doesn't change as the order angle changes. This means the steering system is out of control and is thus very dangerous for vessel. The purpose of building virtual prototyping and conducting fault simulation is to check out those faults that can lead to stuck rudder, in other words, make clear of the influences of all the fault modes to the rudder angle output to see whether they can lead to stuck rudder. On the other hand, other fault modes are also risky in some specific circumstances. For example, fault 15 may lead to collision when the vessel is trying to arrive at port. 


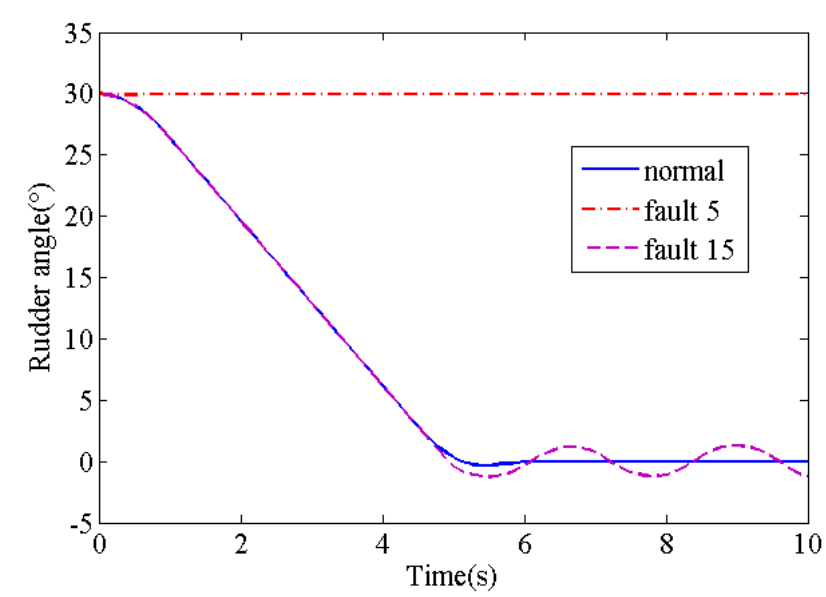

FIGURE IV. CONTRAST OF VIRTUAL PROTOTYPING OUTPUT ANGLE UNDER TWO FAULTY AND NORMAL STATES

\section{REAL-Time FAUlt INJECTION BASED ON CO- SIMULATION}

\section{A. Real-time Fault Injection Approach}

In the simulation duration of AMESim, the parameters of virtual prototyping cannot be modified in real-time as needed. Therefore, real-time fault injection purely in AMESim is impossible.

To resolve this problem, a feasible way is to utilize the Simulink Cosim interface supplied by AMESim. On one hand, this interface uses the arithmetic of AMESim to drive the virtual prototyping. On the other hand, it use MATLAB/Simulink platform to transfer I/O data and thus can modify the parameters of virtual prototyping. In this way, not only the real-time fault injection can be achieved, but also the simulation data is more convenient for processing because of the powerful calculating capacity of MATLAB.

In the following par, one critical problem revolved in the Co-simulation of AMESim and Simulink will be emphasized. That is the input/output relationship between AMESim and Simulink. The right relationship is: The outputs of Simulink are the inputs of AMESim in order and the outputs of AMESim are the inputs of Simulink in reversed order. This relationship is illustrated in Figure 5.

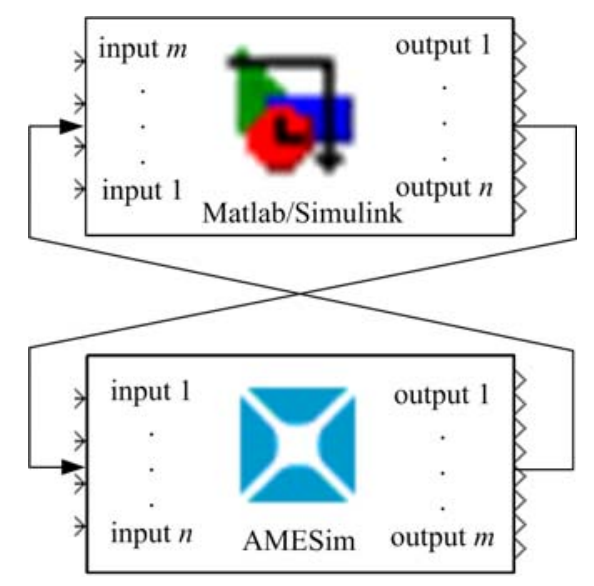

FIGURE V. I/O RELATIONSHIP OF THE INTERFACE

\section{B. Case Study}

Still, fault 5 and fault 15 will be injected in real-time working condition. The fault injection scene is designed as following. The virtual prototyping is in normal condition with a $0^{\circ}$ initial rudder angle when simulation starts. And then, $30^{\circ}$ step order is given to the virtual prototyping. When the system is steady, fault 5 will be injected, after which $0^{\circ}$ step order will be given to see the fault effect. And then, the virtual prototyping will be recovered to normal condition. When the system is steady, fault 15 will be injected, after which $-30^{\circ}$ step order will be given to see the fault effect. The simulation result is illustrated in Figure 6.

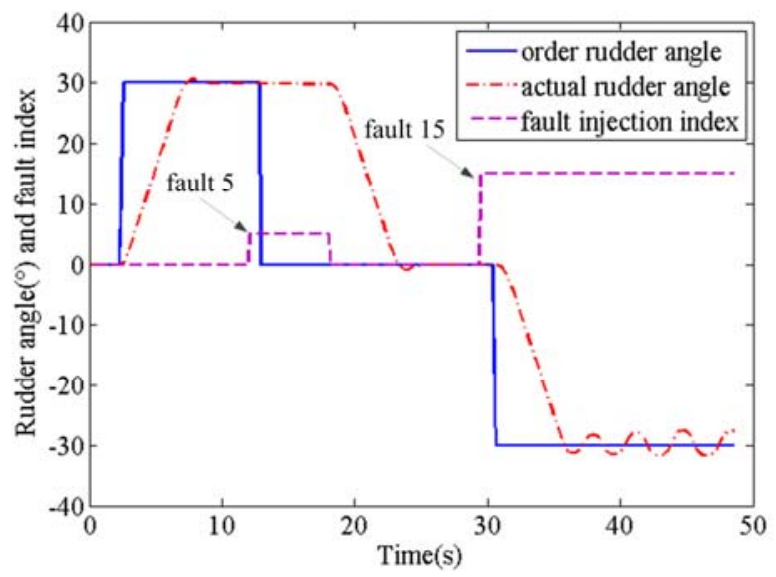

FIGURE VI. REAL-TIME FAULT SIMULATION RESULT

The simulation result showed that faults could be rejected at real-time working condition. After fault 5 was rejected, the actual angle didn't change as the order changed. In other words, fault 5 leads to stuck rudder as studied in section 3.2 with traditional fault injection method. Similar conclusion could be drawn for fault 15. Real-time fault injection and simulation based on Co-simulation of AMESim and Simulink is more flexible and practical than traditional one. 


\section{CONCLUSION}

Simulation based method is a very promising developing direction to deal with the lack of fault data of large-scale mechanic-electronic-hydraulic equipments like steering system. In this paper, virtual prototyping of a steering system was established on AMESim platform and its credibility is validated. On this base, two typical faults were injected in the model. To realize the real-time fault injection, Simulink was utilized to cooperate with AMESim to conduct Co-simulation with Simulink Cosim interface. The simulation results showed that the method proposed in this paper is effective in acquiring realtime fault simulation data. Work in this paper provides an available approach to get fault data in real-time working condition for safety analysis.

\section{ACKNOWLEDGMENT}

This work was supported by the National Natural Science Foundation of China [grant number 51475463].

\section{REFERENCES}

[1] Tingdi Zhao, Safety Design Analysis and Verification, Beijing: National Defense Industry Press; 2011.

[2] F. Xie, J. Wang, Y. Wanga, "Modelling and Co-simulation Based on AMESim andSimulink for Light Passenger Car with Dual State CVT", Procedia Engineering, 16 ( 2011 ), pp.363 - 368.

[3] Deyu He, Niaoqing Hu, MinWang, "Study on Real-Time Fault Injection and Simulation of Mechanic-Electronic-Hydraulic Control System Based on AMESim and LabVIEW", IEEE Prognostics and System Health Management Conference(PHM-2014, Zhangjiajie,China), August 2014, pp. 446-450.

[4] Hu Liangmou, Cao Keqiang, Xu Haojun, "Fault Diagnosis for Hydraulic Actuator Double Closed-loop System Based on Improved LS-SVM", Journal of System Simulation, 2009, 21(17): pp. 5477-5480.

[5] Department of Defense Directive 5000. 61. DoD modeling and simulation verification, validation and accreditation (VV\&A) recommended practices guide. 1996.

[6] Balci O, "Verification, validation, and accreditation of simulation models", Proceedings of the 1997 Winter Simulation Conference, 1997, pp.135-141. 